Optimizing the size and surface properties of polyethylene glycol (PEG)-gold nanoparticles by intense $x$-ray irradiation

This article has been downloaded from IOPscience. Please scroll down to see the full text article.

2008 J. Phys. D: Appl. Phys. 41195301

(http://iopscience.iop.org/0022-3727/41/19/195301)

The Table of Contents and more related content is available

Download details:

IP Address: 140.112.113.225

The article was downloaded on 10/08/2009 at 10:03

Please note that terms and conditions apply. 


\title{
Optimizing the size and surface properties of polyethylene glycol (PEG)-gold nanoparticles by intense $x$-ray irradiation
}

\author{
Chang-Hai Wang ${ }^{1}$, Chi-Jen Liu ${ }^{1}$, Cheng-Liang Wang ${ }^{1}$, Tzu-En Hua ${ }^{1}$, \\ Judy M Obliosca ${ }^{1}$, K H Lee ${ }^{1}$, Y Hwu ${ }^{1,2,3,4,10}$, Chung-Shi Yang ${ }^{5}$, \\ Ru-Shi Liu ${ }^{6}$, Hong-Ming Lin ${ }^{7}$, Jung-Ho $\mathrm{Je}^{8}$ and G Margaritondo ${ }^{9}$ \\ ${ }^{1}$ Institute of Physics, Academia Sinica, Nankang, Taipei 115, Taiwan \\ ${ }^{2}$ Department of Engineering and System Science, National Tsing Hua University, Hsinchu 300, Taiwan \\ ${ }^{3}$ National Synchrotron Radiation Research Center, Hsinchu 300, Taiwan \\ ${ }^{4}$ Institute of Opto-Electronics Sciences, National Taiwan Ocean University, Keelung 202, Taiwan \\ ${ }^{5}$ Nanomedicine Research Center, National Health Research Institute, Hsinchu 350, Taiwan \\ ${ }^{6}$ Department of Chemistry, National Taiwan University, Taipei 106, Taiwan \\ ${ }^{7}$ Department of Materials Engineering, Tatung University, Taipei 104, Taiwan \\ ${ }^{8}$ X-ray Imaging Center, Pohang University of Science and Technology, Pohang 790-784, Korea \\ ${ }^{9}$ Ecole Polytechnique Fédérale de Lausanne (EPFL), CH-1015 Lausanne, Switzerland \\ E-mail:phhwu@sinica.edu.tw
}

Received 14 May 2008, in final form 6 August 2008

Published 5 September 2008

Online at stacks.iop.org/JPhysD/41/195301

\begin{abstract}
The polyethylene glycol (PEG) modified gold nanoparticle complex was synthesized by a one-solution synchrotron x-ray irradiation method. The impact on the structure and morphology of the gold nanoparticles of process parameters such as the PEG molecular weight, the PEG/gold molar ratio and the $\mathrm{x}$-ray dosage were investigated. The size of PEG modified gold particles was found to decrease with increasing PEG addition and x-ray dosage. With the capability to monitor the absorption spectra in situ during the fast synthesis process, this opens the way to accurate control of the size and distribution. PEG chains with an intermediate length (MW6000) were found optimal for size control and colloidal stability in biologically relevant media. Our x-ray synthesized PEG-gold nanoparticles could find interesting applications in nanoparticle-enhanced $\mathrm{x}$-ray tumour imaging and therapy.
\end{abstract}

(Some figures in this article are in colour only in the electronic version)

\section{Introduction}

Nanotechnology is playing an increasingly important role in the fight against cancer. The prospects of nanoparticle-based cancer imaging and therapies have stimulated many studies in this emerging field [1-4]. In particular, gold nanoparticles are promising candidates as carriers for targeted drug delivery $[5,6]$, contrast agents $[7,8]$ or radiotherapy enhancers [9].

The expanding multi-functionalities of gold nanoparticles create challenges for material scientists since additional features such as higher concentration, smaller size, sufficient dispersion and colloidal stability are required for biomedical

10 Author to whom any correspondence should be addressed. applications. This is especially true for in vivo biomedical applications in which, without surface modifications, the administrated nanoparticles are eliminated from circulation within seconds to minutes through the reticulo-endothelial system (RES) [10]. One effective solution to prevent opsonization and enable nanoparticles to circulate for a long time is to construct a 'stealth-shielding' layer composed of water-soluble polymers on the surfaces of particulate vectors [11]. Four well-documented synthetic routes are used to prepare polymer stabilized gold nanoparticles [12]: covalent 'grafting from' (relying on polymerization), covalent 'grafting to' (using sulfur-containing polymers), physisorption and postmodification of pre-formed Au NPs. These methods are summarized in table $1[5,6,8,12,13-29]$. 
Table 1. A summary of preparation approaches for polymer-protected gold nanoparticles.

\begin{tabular}{|c|c|c|c|}
\hline Synthetic route & Advantages & Disadvantages & Reference \\
\hline Covalent graft from & $\begin{array}{l}\text { - Control polymer brush thickness; } \\
\text { - Higher surface polymer graft density }\end{array}$ & $\begin{array}{l}\text { - Multi-steps } \\
\text { - Purification needed }\end{array}$ & {$[13-16]$} \\
\hline Covalent graft to & $\begin{array}{l}\text { - One pot synthesis } \\
\text { - Mono-dispersed Au NPs can be obtained } \\
\text { in aqueous and nonaqueous solutions }\end{array}$ & $\begin{array}{l}\text { - Broad size distribution; } \\
\text { - Long tethered polymer chain unfavourable } \\
\text { for condensation and fractionation } \\
\text { - Sulfur-containing polymer needed }\end{array}$ & {$[17-20]$} \\
\hline Physisorption & $\begin{array}{l}\text { - One pot synthesis } \\
\text { - Unmodified polymer can be used }\end{array}$ & $\begin{array}{l}\text { - Broad size distribution; } \\
\text { - Less stable than covalent bonding; } \\
\text { - A high concentration of protective } \\
\text { agent is required }\end{array}$ & {$[26-29]$} \\
\hline
\end{tabular}

These approaches, however, are affected by limitations that endanger their suitability for specific biomedical application. To achieve maximum effectiveness in enhancing radiotherapy, Au nanoparticles must have optimal size and concentration. Two polymer systems were used: polyethylene glycol (PEG) and poly $N$-vinyl-2-pyrrolidone (PVP). These polymers were successfully exploited to modify nanovectors for gene/drug delivery [30,31], tumour targeting [5, 6, 32, 33] and $\mathrm{x}$-ray imaging $[8,34]$. For the in vivo applications using PEG-gold nanoparticles, all the preparations were based on conventional chemical methods (i.e. sodium citrate or sodium borohydrate reduction) and thiolated PEG [5, 6, 8, 30, 31, 35]. However, the size of gold particles is normally larger than $30 \mathrm{~nm}$. In addition, due to the very low $(\sim 0.25 \mathrm{mM}$ of gold precursor) concentration of colloidal gold, the nanosols must be condensed to reach higher concentration by high speed centrifugation (e.g. $16800 \mathrm{~g}$ for $30 \mathrm{~nm}$ PEG-thiol modified gold nanoparticles [8]). Furthermore, the high cost of thiolated PEG could limit commercial applications.

We report here a one-solution synthesis approach using x-ray irradiation to prepare polymer modified gold nanoparticles that is not affected by the above limitations. The method consists of bombarding a precursor solution containing gold ions and the polymeric stabilizers with intense x-rays produced by a synchrotron source. The irradiation was found to stimulate the formation of gold nanoparticles protected by the polymer chains. Since there is no prior modification of the PEG, the polymeric chains are likely to be physically adsorbed on the particle surfaces. Compared with other physisorption methods, this approach has several advantages: (1) cleanliness, since the system is free of pre-added reducing agents and surfactant; (2) high reproducibility and capability to scale up for mass production; (3) unmodified PEG with low concentration are used; (4) easy increase in concentration with excellent dispersion; (5) high colloidal stability in vivo and in vitro.

The intensity of the x-ray irradiation not only makes it possible to scale up the production, but can be used to accurately control the nanoparticle size.

We investigated in detail the process parameters (molecular weight, polymer concentration and dosage) and their effects on the structure and production rate of PEG modified gold nanoparticles. The mechanism of PEG-gold nanoparticle formation is also discussed. The colloidal stability test confirmed that our high concentration PEG-gold nanosols are stable in typical in vivo and in vitro environments and therefore suitable for possible biomedical applications.

\section{Materials and methods}

\subsection{X-ray synthesis}

Gold nanoparticles were synthesized from a mixed aqueous solution of hydrogen tetrachloroaurate trihydrate $\left(\mathrm{HAuCl}_{4}\right.$. $3 \mathrm{H}_{2} \mathrm{O}$, Aldrich), $\mathrm{NaOH}$ (0.1 M, Showa Inc., Japan) and PEG (Showa Inc., Japan) solutions by bombardment with synchrotron x-ray. The experiments were performed at the BL01A beam line of the NSRRC (National Synchrotron Radiation Research Center) storage rings [36]. Detailed descriptions of the experimental system were previously reported [37-40]. The photon energy distribution was centred between 10 and $15 \mathrm{keV}$ and the dose rate was $5.1 \pm 0.9 \mathrm{kGy} \mathrm{s}^{-1}$ as determined by a Fricke dosimeter with an estimated $G$ value of 13. PEG with different molecular weights $(1500,6000$ and 20000 ) were investigated. The molar ratio PEG/gold ion ranged from 0.004 to 3 . The $\mathrm{pH}$ value of the $1 \mathrm{mM}$ precursor solution was set to 10.3 by adjusting the $\mathrm{NaOH}$ amount and measured with an Orion 720A pH-meter.

The initial PEG-Au colloidal solutions were condensed by combining centrifugation and solvent evaporation under reduced pressure. The centrifugation was performed with an Eppendorf ${ }^{\circledR}$ 5810R, Germany, centrifuge and an Amicon ${ }^{\circledR}$ Ultra-15, Millipore, US centrifugal filter at $4{ }^{\circ} \mathrm{C}$. The centrifugation took place at $3200 \mathrm{~g}$ for $30 \mathrm{~min}$. The gold sols were further condensed by a simple vacuum evaporation system. The final concentration of the PEG-Au colloids was $\sim 75 \mathrm{mg} \mathrm{ml}^{-1}$, as determined by an induced coupled plasma optical emission spectrometer (ICP-OES, Perkin Elmer Optima 3000 DV, Perkin Elmer Co. Ltd, US). 

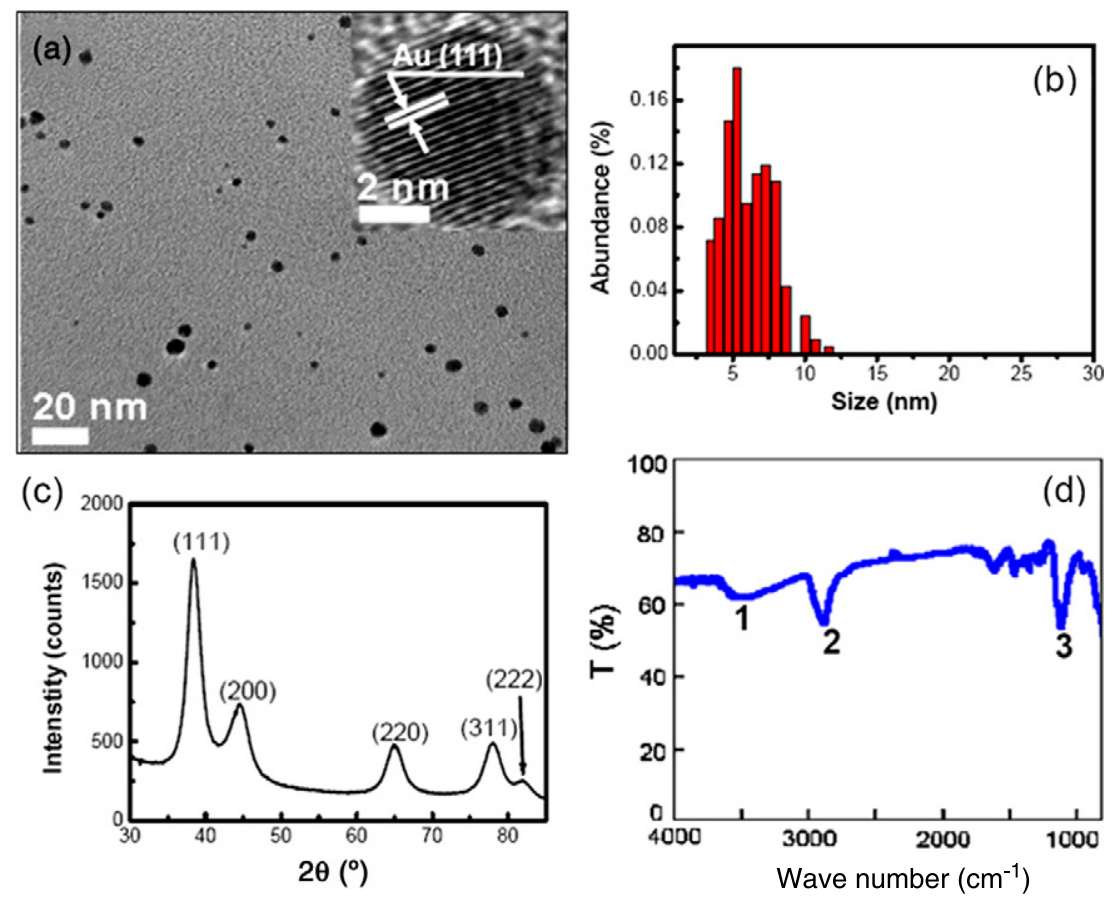

Figure 1. TEM micrograph $(a)$, histogram $(b)$, XRD $(c)$ and FTIR $(d)$ spectra of synchrotron x-ray synthesized PEG-Au nanoparticles in a solution containing $2 \times 10^{-3} \mathrm{M} \mathrm{HAuCl}_{4}$ and $3.3 \times 10^{-5} \mathrm{M}$ PEG (MW6000). The exposure time was $5 \mathrm{~min}$. The HRTEM inset in $(a)$ reveals the $\mathrm{Au}$ (1 111 ) plane of individual nanoparticles.

\subsection{Characterizations}

UV and visible light absorption spectra were taken with a Shimadzu UV-160 spectrometer with a $1 \mathrm{~cm}$ quartz cuvette. Fourier transform infrared spectra of solid PEG-gold samples on a $\mathrm{KBr}$ plate were recorded in the spectral range $4000-500 \mathrm{~cm}^{-1}$ using a JASCO 240 FTIR spectrometer. The surface charge of gold nanoparticle dispersions was measured by a Malvern Zetasizer 3000 HAS (Malvern Instruments Ltd, Malvern, Worcestershire, UK). The dynamic size of the nanoparticles in solutions was measured by dynamic light scattering (DLS) size analyzer (Horiba LB-500, Horiba Co. Ltd, Japan). The particle morphology, structure and size were measured with a JEOL JEM 2010 F field emission gun transmission electron microscope (FEG-TEM) operating at $200 \mathrm{kV}$. The samples for TEM measurements were prepared by placing droplets of nanoparticle-containing solution on carbon-coated $\mathrm{Cu}$ grids and allowing them to dry.

\subsection{Colloidal stability test}

To test the re-dispersion capability, highly concentrated colloid gold was re-dispersed into de-ionized (DI) water to reach an optical density (OD) above 2 . The size and optical absorption of the re-dispersed colloidal gold were then examined by monitoring the ultraviolet-visible (UV-VIS) absorption spectral changes. Gold nanoparticles were also evaluated for their colloidal stability in typical in vitro and in vivo environments. Aliquots of $0.5 \mathrm{ml}$ of re-dispersed condensed gold nanoparticles (OD $>2$ ) were co-cultured with $0.5 \mathrm{ml}$ of different dispersion media including: (1) serumfree culture medium (DMEM); (2) culture medium with $5 \mathrm{wt} \%$ serum; (3) culture medium with $10 \mathrm{wt} \%$ serum; (4) $\mathrm{NaCl}$ (3 M);
(5) BSA (1 mM); (6) HBSS. Samples of the same PEG-Au nanoparticles in $\mathrm{DI} \mathrm{H}_{2} \mathrm{O}$ were used as controls. The colloidal stability was examined by UV-VIS measurement for up to $150 \mathrm{~h}$. Two parameters directly correlated to colloidal stability were used as 'flocculation indicators': (1) the peak maximum and (2) the full width at half maximum (FWHM) of the gold surface plasmon resonance (SPR).

\section{Results and discussion}

\subsection{Characterization of pegylated gold nanoparticles}

TEM micrographs like figure 1(a), taken on our solutions after they dried up, showed that the PEG-Au nanoparticles were spherical with $6.1 \pm 1.9 \mathrm{~nm}$ diameter and reasonable size distribution (figure $1(b)$ ). The inset high-resolution image in figure 1( $a$ ) shows the Au (1 111$)$ plane with a planar spacing of $2.32 \AA$ confirming the crystal structure of the nanoparticles (note that HRTEM cannot reveal the adsorbed PEG because of its low electron density). The crystal nature of PEG-Au particles was also confirmed by x-ray diffraction (XRD) measurements (figure 1(c)). The estimated average nanoparticle size from the broadening of the reflection peak (1 111 ) was $\sim 7.6 \mathrm{~nm}$. The hydrodynamic size measured by DLS for re-dispersed PEG-Au in DI water was $27.9 \pm 8.1 \mathrm{~nm}$. This implies that the thickness of the PEG coating was $\sim 25 \mathrm{~nm}$. A Fourier transform infrared (FTIR) spectrum of PEG-Au is shown in figure $1(d)$. The broad band \#1 in the $3200-3600 \mathrm{~cm}^{-1}$ region is attributed to the $\mathrm{OH}$ group and suggests that hydroxyl plays a role in linking the nanoparticles to the PEG chains. Vibrational bands due to PEG were also detected, including the $\mathrm{CH}_{2}$ group (band $\# 2$ at $2884 \mathrm{~cm}^{-1}$ ) and 
the $\mathrm{C}-\mathrm{O}-\mathrm{C}$ stretching band $\# 3$ at $1114 \mathrm{~cm}^{-1}$ — confirming that PEG chains were immobilized at the nanoparticle surfaces.

Our previous work [37-40] had developed a new approach to prepare highly concentrated colloidal gold solutions free of reducing agents and stabilizers by roomtemperature synchrotron x-ray irradiation. This, as we already mentioned, offers several advantages-quite relevant to potential applications in the biomedical domain. However, biomedical application requires sufficient stability of the particles in vivo-not guaranteed by 'naked' gold nanoparticles. We report here on a development of our approach that solves this crucial problem: by using biocompatible PEG. We can accurately define the size and

Table 2. Influences of PEG molecular weight and PEG/Au ratio on particle size and distribution ${ }^{\mathrm{a}}$.

\begin{tabular}{cclll}
\hline & $\begin{array}{l}\text { PEG chain } \\
\text { length } \\
\text { (Dalton) }\end{array}$ & $\begin{array}{l}\text { PEG/Au } \\
\text { molar } \\
\text { ratio }\end{array}$ & $\begin{array}{l}\text { Particle } \\
\text { size } \\
\text { (mean } \pm \text { SD nm) }\end{array}$ & $\begin{array}{l}\text { Number of } \\
\text { particles } \\
\text { counted }\end{array}$ \\
\hline 1 & 0 & 0 & $20.4 \pm 6.3$ & 186 \\
2 & 1500 & 0.657 & $8.9 \pm 2.9$ & 244 \\
3 & 1500 & 3.285 & $9.2 \pm 3.7$ & 163 \\
4 & 6000 & 0.004 & $10.6 \pm 4.1$ & 253 \\
5 & 6000 & 0.165 & $6.1 \pm 1.9$ & 267 \\
6 & 6000 & 0.325 & $5.4 \pm 1.6$ & 275 \\
7 & 6000 & 1.625 & $4.9 \pm 2$ & 259 \\
8 & 6000 & 2.929 & $5.6 \pm 1.6$ & 287 \\
9 & 20000 & 0.0125 & $8.3 \pm 1.9$ & 219 \\
10 & 20000 & 0.05 & $8.3 \pm 2.0$ & 263 \\
\hline
\end{tabular}

${ }^{\mathrm{a}}$ Reaction condition: $1 \mathrm{mM}$ of $\mathrm{HAuCl}_{4} ; \mathrm{pH}=10.3 ; 5 \mathrm{~min}$ exposure. the surface to meet the requirements of possible practical applications.

\subsection{Effect of the molecular weight and of the PEG/Au ratio}

Both such parameters affect the particle size and distribution of our colloidal. As shown in table 2, only large particles $(8-10 \mathrm{~nm})$ can be obtained when either short (PEG 1500) or long PEG (PEG 20000) chains are included. In contrast, small sizes $(5-7 \mathrm{~nm})$ can be attained when PEG of medium length (PEG 6000) is used. Note in table 2 the sharp size decrease after a small number (PEG/Au $=0.004)$ of PEG molecules are added to the solution: the size $(10.6 \pm 4.1)$ is halved with respect to the unmodified gold particles $(20.4 \pm 6.3 \mathrm{~nm})$.

We also found that gold particle size changed substantially with the PEG density in the solution during irradiation: a larger $\mathrm{PEG} / \mathrm{Au}$ molar ratio resulted in slightly smaller sizes. The particle size saturated at approximately $4-5 \mathrm{~nm}$ after the PEG/Au molar ratio reached 0.17 . The dependence of the size and dispersion of PEG (6000) particles on the PEG density, derived from TEM micrographs, is shown in figure 2. As the PEG density increased (figures $2(a)-(d)$ ), the particle size decreased; at the same time, the size distribution improves and the inter-particle spacing decreased. The TEM results of figure 3(a) show that low weight PEG chains are not sufficient to limit the $\mathrm{Au}$ particle size and some large gold particles coexist with the smaller ones. The Au particles tend to form larger clusters by fusing together. In contrast, high weight PEG chains (PEG 20000) improved the particle dispersion (figure $3(b)$ ).
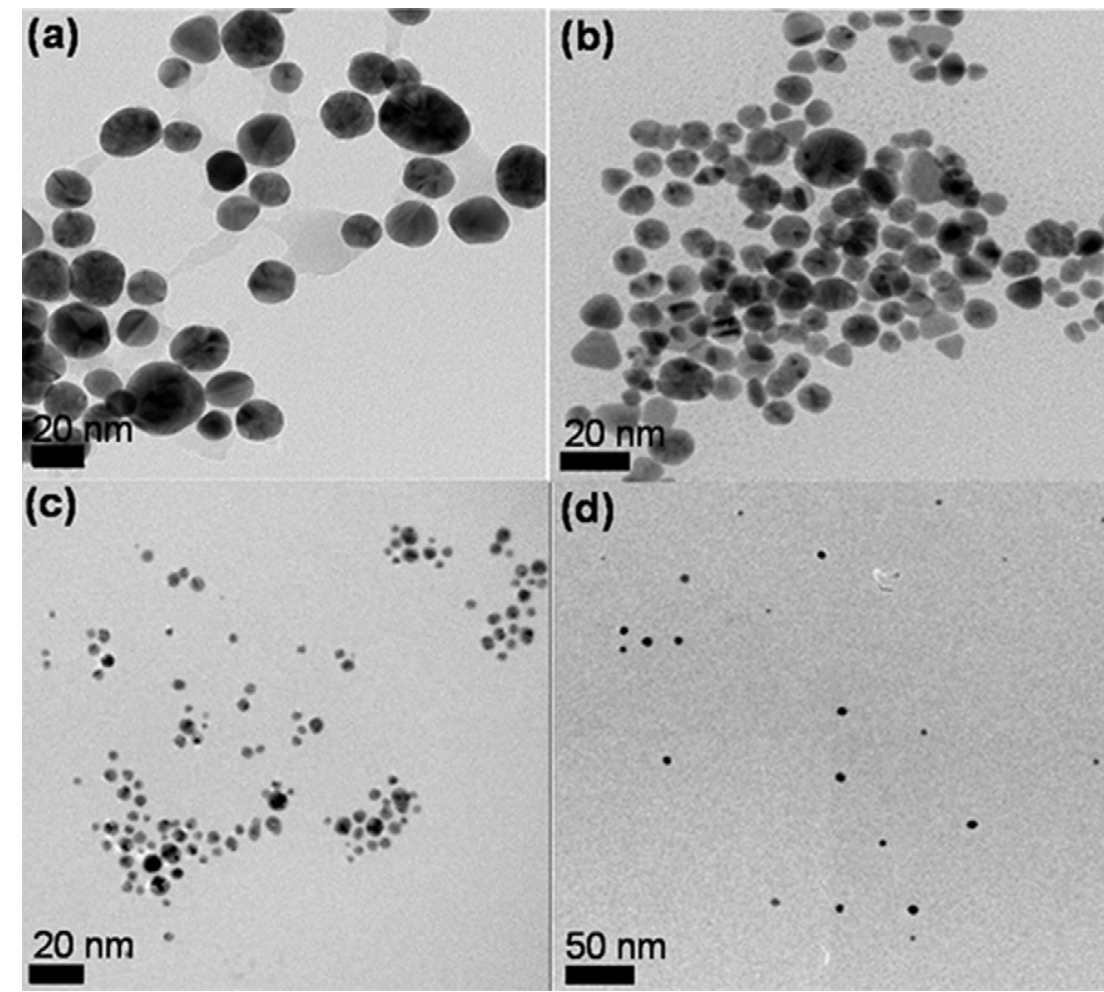

(d)
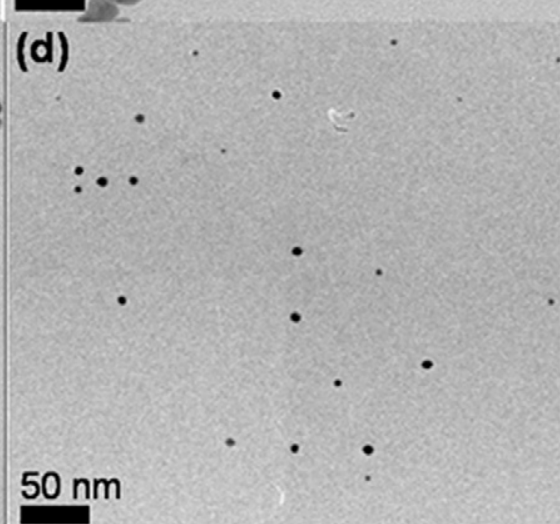

Figure 2. TEM micrographs showing the effects of PEG/Au molar ratio for PEG (MW6000) on the particle size and distribution: (a) 0; (b) 0.004 ; (c) 0.165 and $(d) 1.625$. 


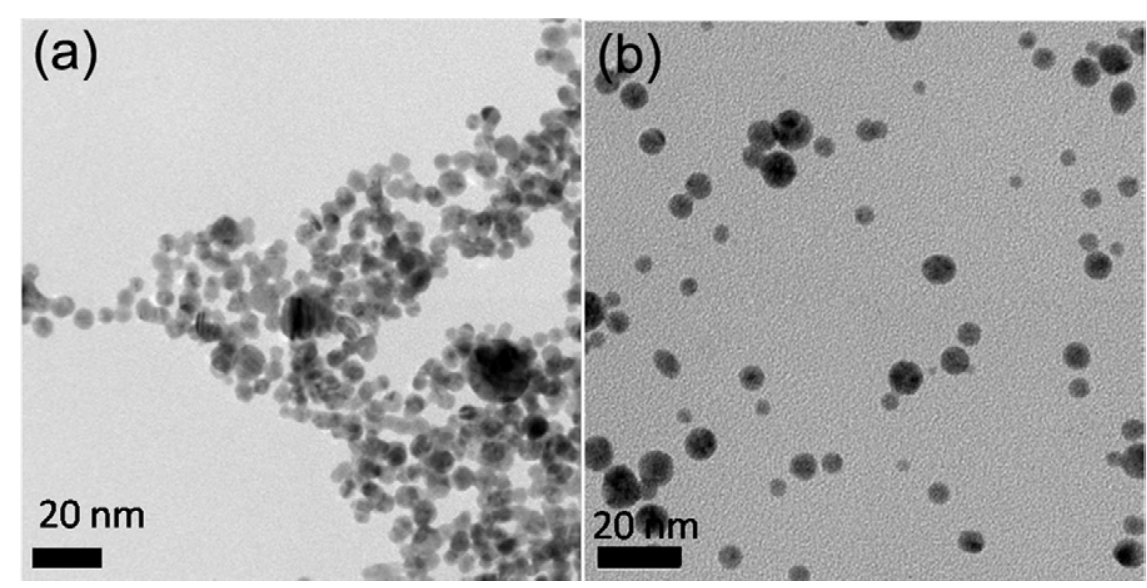

Figure 3. TEM micrograph of PEG-gold nanoparticles showing the effects of molecular weight on the particle morphology and distribution for PEG with different molecular weights; (a) 1500 and (b) 20000.

Thiol-stabilized PEG-gold nanoparticles are a model system in nanotechnology and were therefore the subject of intensive investigations [19, 20,41]. Recently, Shimin et al [19] studied the effects of the size and concentration of PEG-thiol on the gold nanoparticle size. In contrast, only limited literature exists on PEG reduced and stabilized gold nanoparticles [26].

Longenberger and Mills [26] examined the role of PEG in the formation of gold colloid in air-saturated aqueous solution systems. They concluded that the oxidation of the oxylene groups leads to the reduction of gold ions and that an increase in the PEG molar mass increases the particle production rate. However, only large nanoparticles were obtained and the gold colloidal tended to flocculate and agglomerate. Furthermore, since PEG is a weak reducing agent, to obtain stable colloidal gold, a PEG/Au molar ratio as high as 30 and a long co-culture time $(>8 \mathrm{~h}$ ) were required [26].

In our $\mathrm{x}$-ray irradiation approach, the PEG/Au ratio is very small (e.g. 0.167 for typical PEG 6000-gold preparation) and the role of PEG macromolecules is to control the size and to stabilize the gold nanoparticles within the PEG chains. We did observe that both the polymer molecular weight and the PEG/Au ratio affected the Au particle size and their dispersion. We found that neither very low nor very high weight PEG chains are effective in limiting the Au particle size: the minimal size is produced by a PEG molecular weight around MW6000. The PEG molecules are unlikely to covalently bond to gold, thus neutral PEG molecules are expected to be physisorbed on the gold surfaces. The action of PEG chains is closely related to their affinity to $\mathrm{AuCl}_{4}^{-}$ions and conformation on the initially formed gold clusters. However, the details of the PEG chainsAu nanoparticle interaction in our synthesis require additional investigations.

For PEG 6000, as the PEG/Au ratio increased from 0.004 to 0.1645 , the gold particle size decreased from $10.6 \pm 4.1$ to $4.9 \pm 2 \mathrm{~nm}$. This could be due to the increase in surface density of PEG molecules immobilized on the Au particles. This is consistent with the fact that as the PEG/Au ratio increases further the particle size remains unchanged-suggesting the saturation of the PEG coverage of the Au surfaces. The criterion for selecting the PEG/Au ratio 0.167 for more detailed studies was to achieve a small Au size with minimum PEG loading. In fact, excessive PEG negatively impacts characterization and condensation [26].

\subsection{Effects of the exposure time}

These effects on the morphology and size dispersion of PEGgold nanoparticles are shown in figure 4. For PEG with different chain lengths (6000 and 20000), the trend is that as the exposure time increases the initially formed Au clusters disintegrate and smaller particles with improved dispersion were formed. As shown in figure 4(a), after an x-ray exposure of $30 \mathrm{~s}$, PEG-gold clusters interconnected/fused together leading to an overall size above $100 \mathrm{~nm}$ in the network structure. After further exposure for 5 min (figure $4(b)$ ), well-dispersed PEG-gold nanoparticles with size $<7 \mathrm{~nm}$ were obtained. During an additional exposure for up to $15 \mathrm{~min}$ (figure $4(c)$ ), the size and particle morphology remained unchanged. For PEG 20000 (figures $4(d)-(f)$ ), the exposure time effects were similar to PEG 6000 but with some noteworthy differences. For example, after an exposure of $30 \mathrm{~s}$ bigger gold nanoparticle $(15-20 \mathrm{~nm})$ were formed instead of the inter-connected structure. Additional x-ray irradiation led to a decrease in the particle size to approximately $8 \mathrm{~nm}$ (figure $4(f)$ ).

The dependences of gold particle size and morphology on exposure time (3-900s) can be linked to the evolution of optical absorption spectra. As shown in figure 5(a), for PEG-gold nanoparticles formed after a $10 \mathrm{~s}$ exposure, only a very broad absorption peak centring at approximately $542 \mathrm{~nm}$ is observed indicating the larger cluster size and small yield of colloidal gold. These colloids are not stable and serious flocculation occurs and precipitates formed. At exposure time longer than $1 \mathrm{~min}$, the characteristic SPR peak of colloidal gold appears at $509 \mathrm{~nm}$. Using the intensity of the SPR peak as an indicator of the colloidal gold concentration, as shown in figure $5(b)$, we found that almost all gold precursor ions have been reduced to colloidal gold after 2-3 min of 


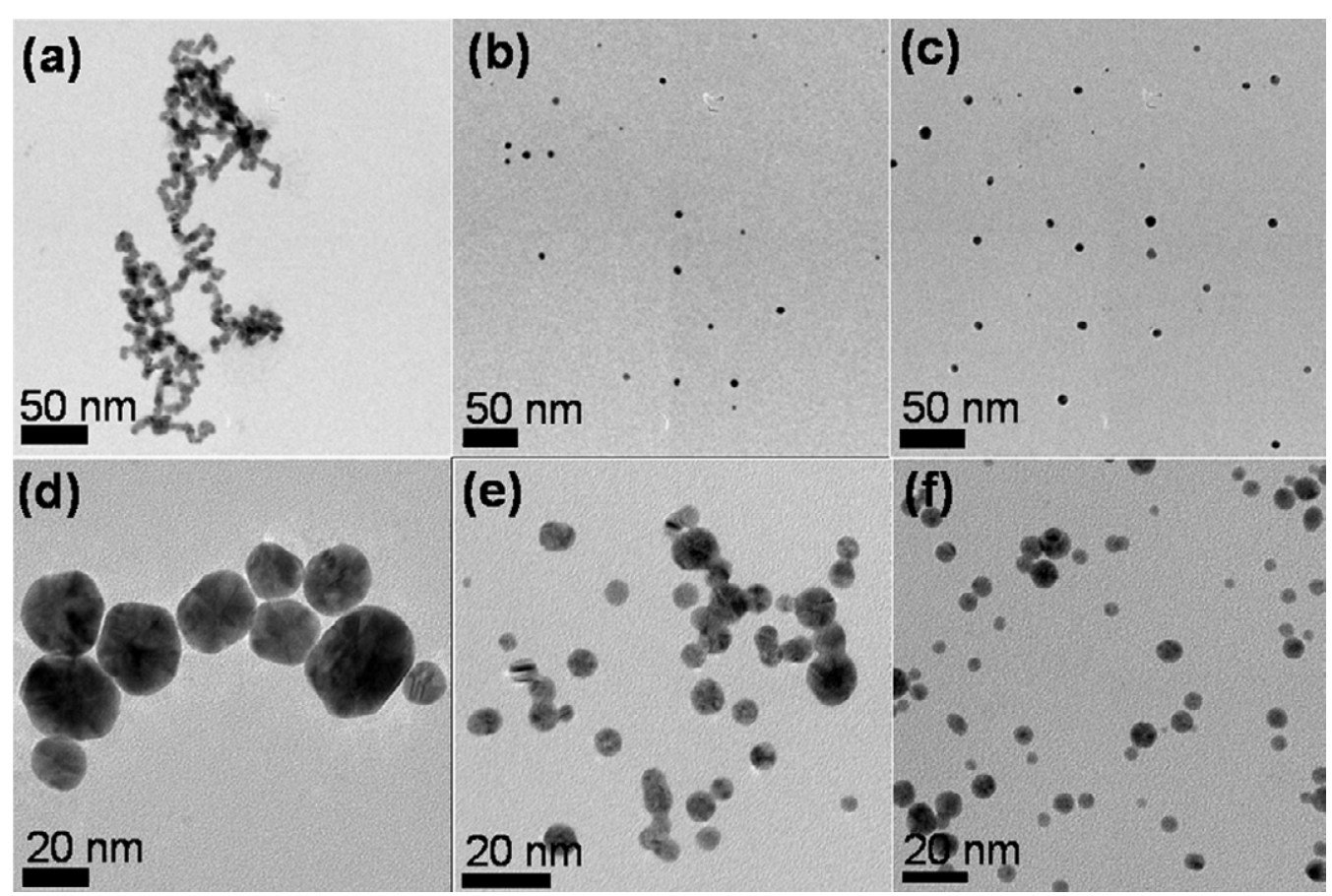

Figure 4. TEM micrographs showing the effects of x-ray dosage on gold particle size, distribution and morphology. PEG 6000: (a) 30 s, (b) $5 \mathrm{~min}$ and (c) $15 \mathrm{~min}$; PEG 20000: (d) $30 \mathrm{~s}$; (e) $1.5 \mathrm{~min}$ and $(f) 5 \mathrm{~min}$.
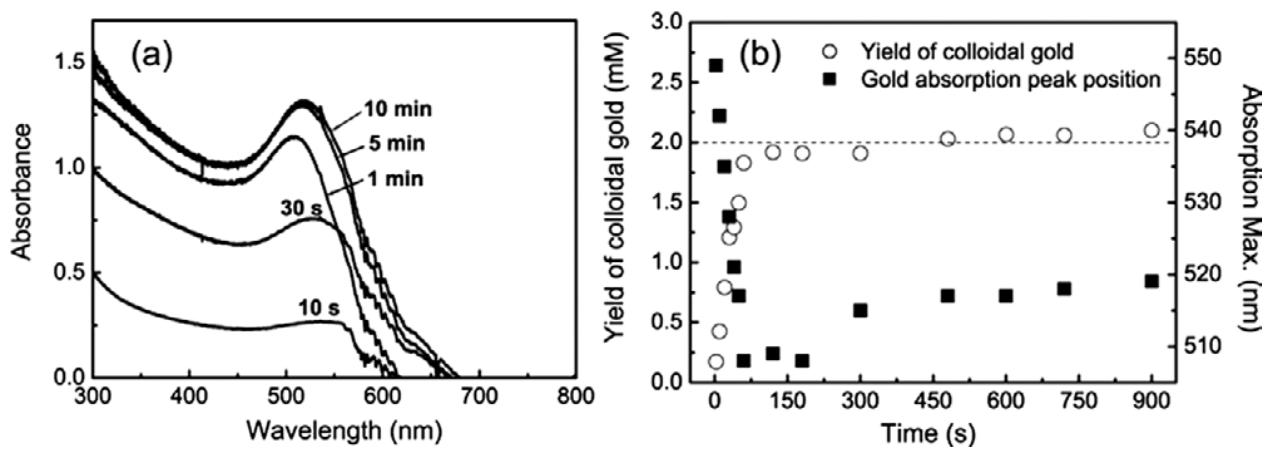

Figure 5. Visible optical absorption of PEG-Au nanosols prepared with various exposure times: $(a)$ absorption spectra and $(b)$ the variation of colloidal yield and gold SPR peak position.

exposure. This fast reducing speed and the $\sim 100 \%$ reduction efficiency adds to the advantage of our x-ray irradiation approach.

It was also observed that the SPR peak position depends sensitively on the exposure time (figure $5(b)$ ). The SPR peak firstly blue shifts up and then reversely shifts to longer wavelength with $\mathrm{x}$-ray exposure time longer than $1 \mathrm{~min}$. Normally the SPR peak red shifts with increasing particle size. The blue shift at exposure time shorter than $1 \mathrm{~min}$ is consistent with this argument. However, the following red shift is inconsistent with the TEM observation that the particle size decreases upon longer exposure. The temperature effect (the solution temperature increases approximately $13{ }^{\circ} \mathrm{C}$ after $5 \mathrm{~min}$ exposure) also predicts a blue shift [42]. We can rule out the possible influence of variation in inter-particle displacement since mono-dispersed gold nanosols are obtained (figure 1(a)). Further investigations are required to explain the observed red shift of the SPR peak.

\subsection{Colloidal stability}

Stability tests were performed on re-dispersed nanoparticles for up to $150 \mathrm{~h}$ using UV-VIS absorption spectra, as shown in figure 6. Specifically, good colloidal stability was demonstrated by the stability of the SPR peak. This conclusion is valid for all dispersion media we explored except DMEM. In the latter case (figure 6(a)), after $100 \mathrm{~h}$ an additional absorption feature appeared at $585 \mathrm{~nm}$ that could be due to flocculation. The absorption spectra in figures $6(b)-(d)$ reveal instead excellent stability for the dispersion media containing HBSS, BSA and $\mathrm{NaCl}(3 \mathrm{M})$.

Quantitatively speaking, the stability can be characterized in terms of the time variations of the so-called 'flocculation indicators': the SPR peak FWHM and peak position. The results, summarized in table 3, confirm the qualitative message of figure 6, i.e. high stability for BSA (1 mM) and HBSS media, good stability for the $\mathrm{NaCl}$ medium and somewhat lower 

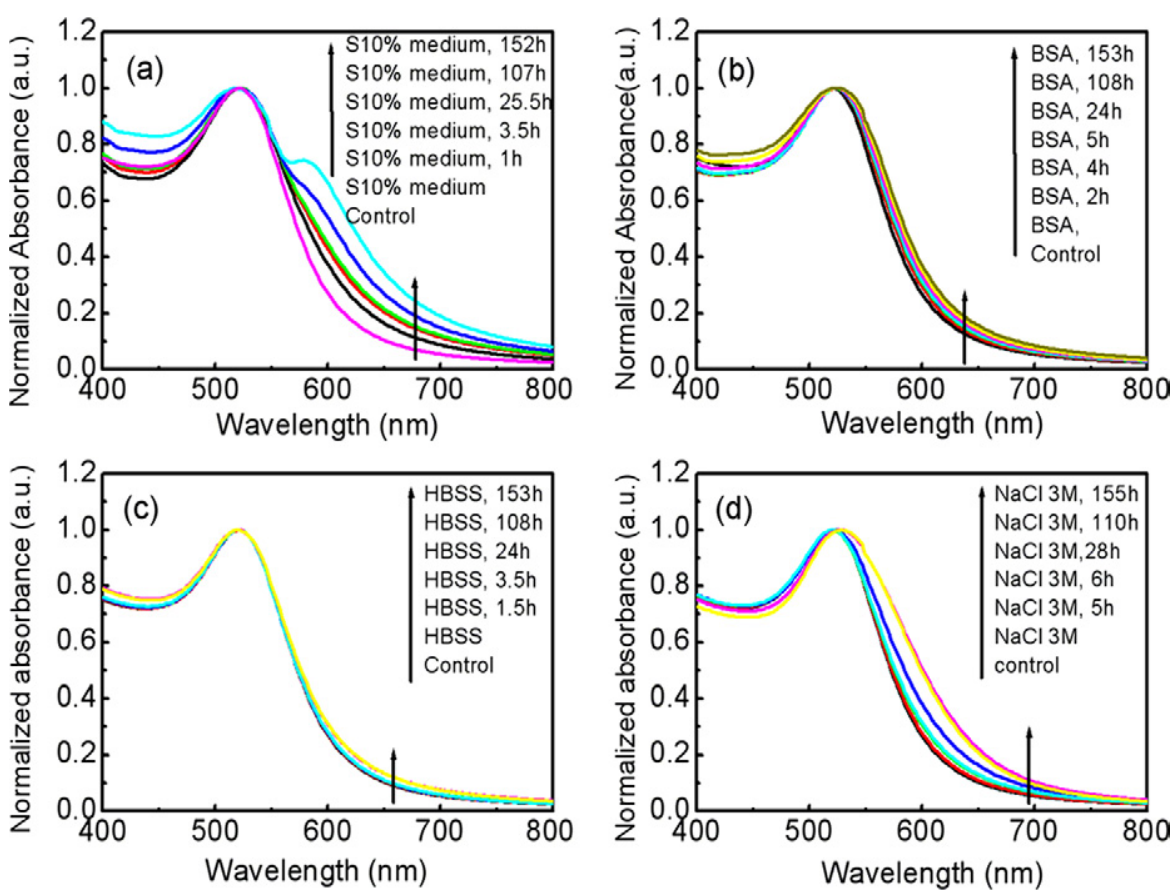

Figure 6. UV-VIS spectra of re-dispersed PEG-Au colloid in different dispersion media: (a) DMEM containing 10\% serum; $(b)$ BSA; (c) $\mathrm{HBSS}$ and $(d) \mathrm{NaCl}(3 \mathrm{M})$.

Table 3. Time-dependent colloidal stability of re-dispersed PEG-Au colloid in different media.

\begin{tabular}{|c|c|c|c|c|c|c|c|c|}
\hline \multirow{2}{*}{$\begin{array}{l}\text { Dispersion medium } \\
\text { time point }(\mathrm{h})\end{array}$} & \multicolumn{2}{|c|}{ HBSS } & \multicolumn{2}{|c|}{ BSA } & \multicolumn{2}{|c|}{$\mathrm{NaCl}$} & \multicolumn{2}{|c|}{ DMEM/F12 } \\
\hline & $A_{\max }{ }^{\mathrm{a}}$ & FWHM $^{\mathrm{b}}$ & $A_{\max }$ & FWHM & $A_{\max }$ & FWHM & $A_{\max }$ & FWHM \\
\hline 0 & 82.3 & 521.4 & 523.4 & 85.1 & 521.4 & 83.5 & 521.4 & 84.7 \\
\hline 1 & 81 & 521.4 & 523.4 & 81 & 521.4 & 83.5 & 521.4 & 88.3 \\
\hline 3.5 & 81.5 & 519.4 & 523.4 & 86.6 & 519.4 & 83.5 & 521.4 & 87 \\
\hline 25.5 & 83.5 & 519.4 & 523.4 & 89.1 & 521.4 & 86.1 & 521.4 & 99.5 \\
\hline 107 & 81.8 & 521.4 & 523.4 & 91.4 & 527.4 & 100 & 517.4 & 130.1 \\
\hline 152 & 83.5 & 521.4 & 523.4 & 94.9 & 529.4 & 98.7 & 513.4 & 139 \\
\hline
\end{tabular}

a Absorption maximum at Au SPR.

${ }^{\mathrm{b}}$ Full width at half maximum.

stability for the DMEM medium. The colloidal stability of PEG-gold nanoparticles was also examined with zeta potential measurements. The zeta potential for the unmodified gold colloidal was $-57.8 \pm 5 \mathrm{mV}$. For the PEG-gold colloidal, the zeta potential decreased to $-20.1 \pm 7.5 \mathrm{mV}$ at a $\mathrm{pH}$ value of 7.23: this can be attributed to the shielding effect of the neutral PEG chains immobilized on the particle surfaces.

Pegylation is well known to improve the colloidal stability of nanoparticles both in vitro and in vivo. Synchrotron x-ray derived PEG-gold nanoparticles are highly stable even at low polymer concentrations and show no sign of flocculation or aggregation even after one year of storage at ambient temperature. We found that such nanosols are stable under typical in vitro and in vivo conditions for at least $150 \mathrm{~h}$. The stability in different dispersion media decreased according to the sequence: HBSS $>$ BSA $(1 \mathrm{mM})>\mathrm{NaCl}(3 \mathrm{M})>$ cell culture medium (DMEM).

The colloidal stability of PEG-gold nanoparticles could be explained by the steric repulsion of the nanoparticles due to the presence of PEG. We also found that PEG-Au is influenced much more by the adsorbed serum component than by the ionic strength of the dispersion media, demonstrating steric stabilization dominates over electrostatic stabilization. Under typical in vivo conditions with high ionic strength, PEGgold nanosols exhibited superior colloidal stability whereas unmodified gold nanoparticles rapidly flocculated and formed sediments. The critical coagulation concentration (CCC) of PEG-gold determined by UV-VIS spectra was $>2.5 \mathrm{M}$ (whereas for unmodified gold it was $>0.025 \mathrm{M}$ ). The steric repulsion due to hydrophilic PEG molecules limited the size of the nanoparticles and resulted in good dispersion.

As shown in figure 6, the stability of PEG-gold nanosols in the DMEM medium gradually decreased as the incubation time increased. Serum adsorption on the surfaces of the PEG-Au particles is likely to contribute to this phenomenon. Serum protein adsorption on PEG-Au particles was revealed by directly examining the hydrodynamic size of the particles loaded into the cell medium. The DLS measurement detected a larger size, typically $>100 \mathrm{~nm}$, for PEG-Au nanoparticles in DMEM medium compared with the control system (around $29.8 \mathrm{~nm}$ ). The stabilizing role of PEG depends on different factors such as the chain length, the inter-chain separation 
and the PEG surface density $[19,43]$. Therefore, the timedependent colloidal stability of PEG-Au is probably due to the incomplete coverage by immobilized PEG chains on the gold surfaces.

\section{Conclusion}

We developed a one-solution x-ray irradiation approach to prepare PEG modified gold nanoparticles using pristine PEG molecules. A low concentration of unmodified PEG macromolecules was very effective in controlling particle size and in stabilizing the gold nanoparticles. Both the PEG chain length and the PEG/Au ratio affected the size, morphology and colloidal stability of the particles. The PEGgold nanosols could be easily concentrated and re-dispersed and demonstrated high stability under realistic biomedical conditions.

\section{Acknowledgments}

This work was supported by the National Science Council, the Academia Sinica (Taiwan), the Creative Research Initiatives (Functional X-ray Imaging) of MOST/KOSEF (Korea), the Center for Biomedical Imaging (CIBM) in Lausanne, partially funded by the Leenaards and Jeantet foundations and by the Swiss Fonds National de la Recherche Scientifique and by the EPFL.

\section{References}

[1] Zharov V P, Galitovskaya E N, Johnson C and Kelly T 2005 Lasers Surgery Medicine 37219

[2] Ferrari M 2005 Nature Rev. Cancer 5161

[3] El-Sayed I H, Huang X H and El-Sayed M A 2006 Cancer Lett. 239129

[4] Caruthers S D, Wickline S A and Lanza G M 2007 Curr. Opin. Biotechnol. 1826

[5] Paciotti G F, Myer L, Weinreich D, Goia D, Pavel N, Mclaughlin R and Tamarkin L 2004 Drug Deliv. 11169

[6] Paciotti G F, Kingston D G I and Tamarkin L 2006 Drug Develop. Res. 6747

[7] Hainfeld J F, Slatkin D N, Focella T M and Smilowitz H M 2006 Br. J. Radiol. 79248

[8] Kim D K, Park S J, Lee J H, Jeong Y Y and Jon S Y 2007 J. Am. Chem. Soc. 1297661

[9] Hainfeld J F, Slatkin D N and Smilowitz H M 2004 Phys. Med. Biol. 49 N309

[10] Owens D E III and Peppas N A 2006 Int. J. Pharm. 30793

[11] Vlerken L E V, Vyas T K and Amiji M M 2007 Pharm. Res. 241405

[12] Shan J and Tenhu H 2007 Chem. Commun. 4580

[13] Kotal A, Mandal T K and Walt D R 2005 J. Polymer Sci. A 433631
[14] Zhao H, Kang X and Liu L 2005 Macromolecules 3810619

[15] Raula J, Shan J, Nuopponen M, Niskanen A, Jiang H, Kauppinen E and Tenhu H 2003 Langmuir 193499

[16] Zhao W, Gao Y, Kandadai S A, Brook M A and Li Y 2006 Angew. Chem. Int. Edn $\mathbf{4 5} 2409$

[17] Corbierre M K, Cameron N S and Lennox R B 2004 Langmuir 202867

[18] Hussain I, Graham S, Wang Z, Tan B, Sherrington D C, Rannard S P, Cooper A I and Brust M 2005 J. Am. Chem. Soc. 12716398

[19] Shimin R G, Schoch A B and Braun P V 2004 Langmuir 205613

[20] Sakura T, Takahashi T, Kataoka K and Nagasaki Y 2005 Colloid Polymer Sci. 28497

[21] Kang Y and Taton T A 2005 Macromolecules 386115

[22] Becker C F W, Marsac Y, Hazarika P, Moser J, Goody R S and Niemeyer C M 2007 ChemBioChem 832

[23] Zubarev E R, Xu J, Sayyad A and Gibson J D 2006 J. Am. Chem. Soc. 1284958

[24] Ujihara M, Mitamura K, Torikai N and Imae T 2006 Langmuir 223656

[25] Shenoy D, Fu W, Li J, Crasto C, Jones G, Dimarzio C, Sridhar S and Amiji M 2006 Int. J Nanomedicine 151

[26] Longenberger L and Mills G 1995 J. Phys. Chem. 99475

[27] Ah C S, Yun Y J, Park H J, Kim W J, Ha D H and Yun W S 2005 Chem. Mater. 175558

[28] Hoppe C E, Lazzari M, Pardinas-Blanco I and Lopez-Quintela M A 2006 Langmuir 227027

[29] Zhou M, Chen S and Zhao S 2006 J. Phys. Chem. B 1104510

[30] Kawano T, Yamagata M, Takahashi H, Niidome Y, Yamada S, Katayama Y and Niidome T 2006 J. Control. Release 111382

[31] Bergen J M, Recum H A V, Goodman T T, Massey A P and Pun S H 2006 Macromol. Biosci. 6506

[32] Oyewumi M O, Yokel R A, Jay M, Coakley T and Mumper R J 2004 J. Control. Release 95613

[33] Liu Z, Cai W B, He L, Nakayama N, Chen K, Sun X M, Chen X Y and Dai H J 2007 Nature Nanotechnol. 247

[34] Rabin O, Perez J M, Grimm J, Wojtkiewicz G and Weissleder R 2006 Nature Mater. 5118

[35] Kawano T, Katayama Y and Niidome Y 2006 J. Control. Release 114343

[36] Margaritondo G, Hwu Y and Je J H 2004 Riv. Nuovo Cimento 277

[37] Kim C C, Wang C H, Yang Y C, Hwu Y, Seol S K, Kwon Y B, Chen C H, Liou H W, Lin H M, Margaritondo G and Je J H 2006 Mater. Chem. Phys. 100292

[38] Yang Y C, Wang C H, Hwu Y and Je J H 2006 Mater. Chem. Phys. 10072

[39] Wang C H et al 2007 Mater. Chem. Phys. 106323

[40] Wang C H, Chien C H, Yu Y L, Liu C J, Lee C F, Chen C H, Hwu Y, Yang C H, Je J H and Margaritondo G 2007 J. Synchrotron Radiat. 14477

[41] Liu Y L, Shipton M K, Ryan J, Kaufman E D and Franzen S 2007 Anal. Chem. 792221

[42] Stephan L and Mostafa A E S 1999 J. Phys. Chem. B 1034212

[43] Gref R, Lück M, Quellec P, Marchand M, Dellacherie E, Harnisch S, Blunk T and Mülle R H 2000 Colloids Surf. B 18301 